\title{
EVALUATION OF A CRICKET BOWLING MACHINE WITH AN ARM AND HAND TO DELIVER THE BALL.
}

\author{
Ronnie Bickramdass ${ }^{1 *}$, Prakash Persad ${ }^{2}$, Kelvin Loutan $\mathrm{Jr}^{3}$ and \\ Aaron Ameerali ${ }^{4}$ \\ ${ }^{1,2,4}$ Design and Manufacturing, The University of Trinidad and Tobago, Trinidad \\ ${ }^{3}$ Faculty of Engineering and Physical Sciences, University of Surrey, England \\ ${ }^{1}$ Email: ronnie.bickramdass@utt.edu.tt*(Corresponding author) \\ ${ }^{2}$ Email: Prakash.persad@utt.edu.tt \\ ${ }^{3}$ Email: k.loutan@surrey.ac.uk \\ ${ }^{4}$ Email: aaron.ameerali@utt.edu.tt
}

\begin{abstract}
The use of bowling machines to train batsmen whether it be indoors or outdoors have increased significantly. In the absence of bowlers, batsmen can bat for hours without any bowlers getting tired. The designs of these machines are often a derivative of ball projection machines used for other sports such as tennis. Reviewed literature highlights the deficit in visual information in the form of an arm and hand when using these machines. Hence a cricket bowling machine was developed with an arm and hand. The usability, functionality, repeatability and accuracy of the cricket bowling machine with an arm and hand was tested which had been previously designed and built by Dr Kelvin Loutan Jr (2016) at The University of Trinidad and Tobago. A trajectory model was developed for an indoor environment and experimentally validated with data collected from extensive testing of the bowling machine using Pitch Vision hardware and software. A design procedure had to be formulated to determine what test had to be done and the method of collecting data. The testing, collecting data and validation of the model was done with the cricket bowling machine at its current state with minor changes to the hand. The release angle at which the ball leaves the hand was found to have a significant impact on the length of the delivery. Finally, the bowling machine was able to bowl various lengths and varying speeds consistently. The variation in speed placed the machine in the category of mediumfast, that is, speeds between $120 \mathrm{~km} / \mathrm{h}(75 \mathrm{mph})$ and $130 \mathrm{~km} / \mathrm{h}(81 \mathrm{mph})$.
\end{abstract}

Keywords: Arm and hand, testing procedure, collecting data, repeatability and validation https://doi.org/10.47412/VCAQ2784

\section{Introduction}

Cricket has three (3) major aspects in batting, bowling and fielding. Bowling is one of the most difficult tasks in the game of cricket and bowlers tend to save themselves for the actual match and conserve in practice to avoid injuries. During batting practice, batsmen are drilled in facing deliveries that are particularly difficult. For this, bowlers are made to repetitively bowl thus allowing the batsmen to become attune to the bowling action. Bowling machines are thus used at clubs and academies to facilitate this repetition. Bowling machines also provides solo training in the absence of a bowler.

The cricket bowling machine permits batsmen to practice for hours but tends to develop some bad practices in the batsman set up and trigger movement. The trigger movement is the initial step the batsman does before striking the ball. A coach would want the batsman, in training, to bat for hours and to set up exactly 
as in a match. The traditional machines do not allow for this practice as the coach or assistant must give a signal to the batsman before feeding the ball in to the machine. The batsman would normally tend to change their set up to facilitate the machine. Hence the rational for the development of a bowling machine with the arm and hand.

The current bowling machine shoots the ball out by various means which prevents the batsman from tracking the ball before being delivered, therefore the batsman is unable to anticipate the point where the ball pitches and the bounce after pitching. A batsman facing traditional bowling machines would usually change their batting style such as, earlier backswing, earlier downswing and even shorter stride towards the ball. The Bowling arm and hand is the main visual cue for highly skilled batsmen. When using traditional bowling machines, a visual cue must therefore be given to the batsman before the ball is fed into the machine. The traditional bowling machine is unable to bowl the leather ball that is used in matches. It uses a poly soft ball especially design for those machines with the wheels.

There are several types of ball projection machines which are used as a coaching aid in sports such as tennis, baseball and cricket. These machines fall into one of the following categories; pneumatic cannons, catapult mechanisms and counter rotating wheeled machines. The Kanon Bowling Machine, manufactured by Howard Manufacturing in South Africa, is the only pneumatic cricket bowling machine around. The counter rotating wheel type bowling machines consist of a single wheel type, two (2) wheel type, three (3) wheel type and four (4) wheel type machines. The more popular machines are the two wheels and three wheels types. Some common brands of the wheel type machines are Flicx, Bola, Winters, JUGS, Leverage, Merlyn, Slider, Paceman, Deuce and Heater.

The Catapult type bowling machine or Arm type bowling machine utilizes a combination of a spring and motor. The length of the delivery is adjusted by tilting of the machine. A Patent was filed on March 2013, Bangalore India. The ProBatter Video Pitching Machine developed by ProBatter Sports LLC, was establish in 1999 as a baseball pitching machine. In 2010 the machine was adapted for cricket bowling. The system comprises of a conventional bowling machine hidden behind an 8' by 10 ' screen with a hole for passage of the ball. A video of an entire bowler's delivery from run up to ball release, recorded from the batsman's perspective, is projected on the screen. There is also the TrueMan Bowling Simulator developed by Bola which is also utilizes video projection. The Robotic Bowling Arm prototype was developed during the academic year 2005/2006 by Mr. Neil Ramdass, under the supervision of Prof. Prakash Persad, designed, built and tested a working prototype of a robotic bowling arm with hand, at the University of the West Indies. 


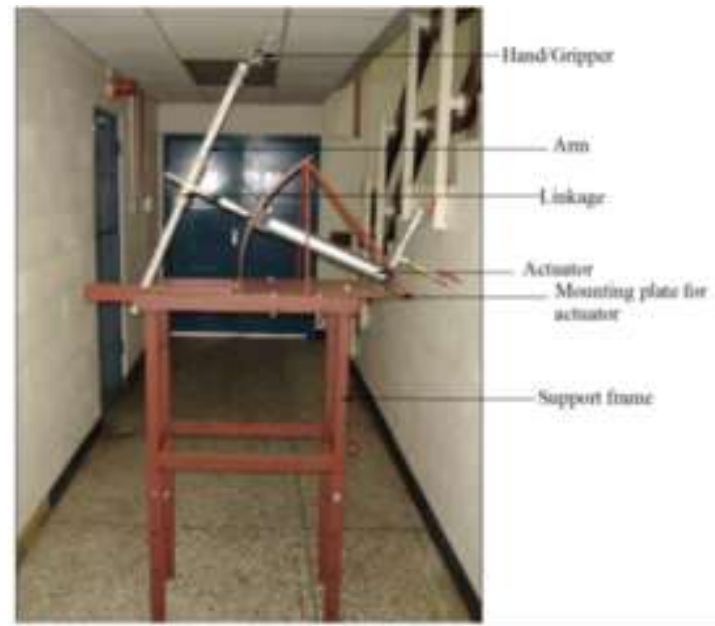

Figure 1: Working bowling arm prototype (Persad \& Ramdass, 2006).

A team of Mechanical Engineering final year students at the University of Adelaide developed a bowling machine prototype. It consists of a vertical frame which supports a rigid bowling arm with a hand. A linear spring is connected to the frame and the lower end of the arm and acts as the actuator of the arm.

The bowling machine used for the testing, in this project, was an Anthropometric Fast Bowling Machine with an arm, developed at The University of Trinidad and Tobago by Dr Kelvin Loutan and supervised by Professor Prakash Persad.

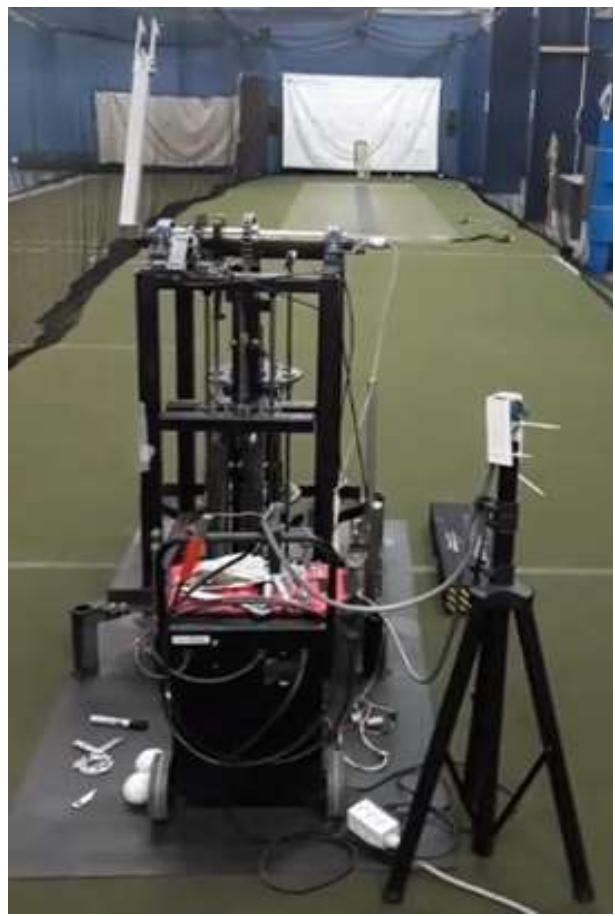

Figure 2: An Anthropometric Fast Bowling Machine (Persad \& Loutan Jr, 2016). 


\section{Machine Design}

Dieter (2000) generalises the engineering design process to 8 stages starting with problem definition and ending with detailed design. This methodology is appropriate for the design of individual components or a system comprising of components of a similar type. One such methodology is that proposed by Puig, Rodriguez and Ceccarelli (2008) for an anthropometrically dimensioned mechatronic device. This design process was thus adopted for the design of the cricket fast bowling machine with the problem being defined as a lack of pre-release data associated with existing bowling machines. The design functions of the proposed machine was identified to be:

- Deliver a cricket ball at fast bowling speeds $(88+\mathrm{mph})$.

- Deliver a cricket ball via an arm with hand.

- Be anthropometrically dimensioned

The operational steps of the machine were developed, and a functional flow chart was obtained.

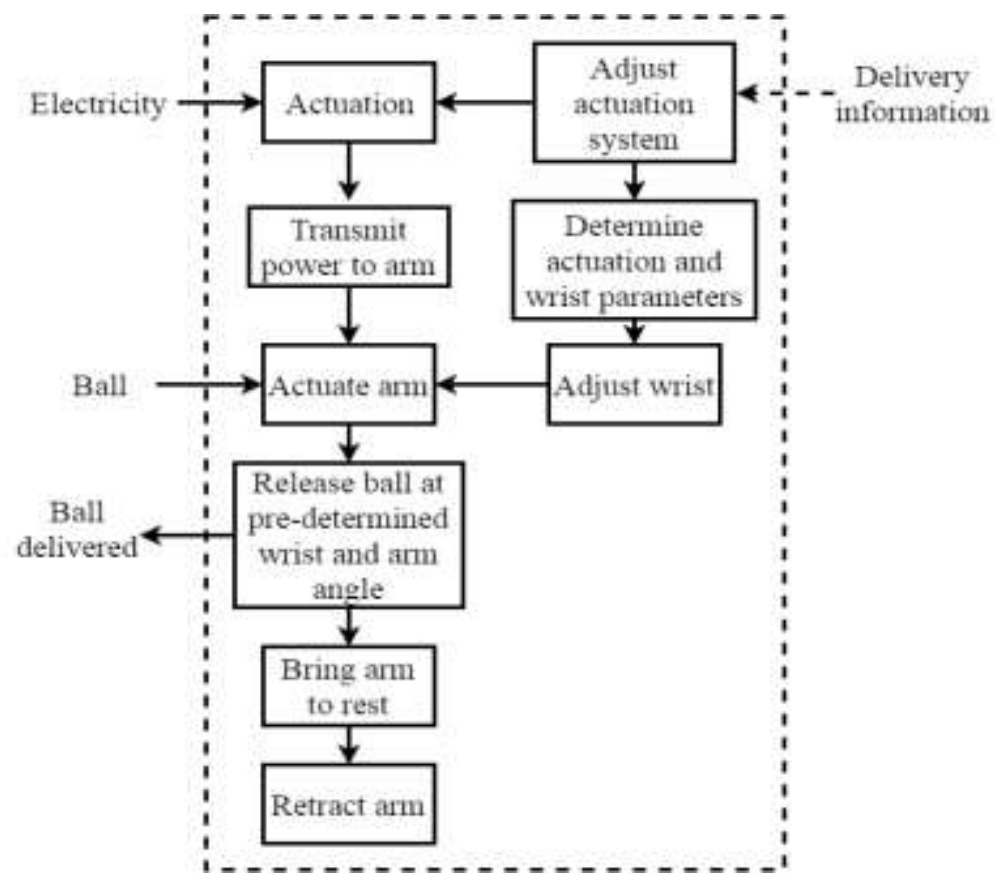

Figure 3: Functional flow chart

To establish the general dimensions of the machine, the biomechanics of a fast bowler's delivery was reviewed. During the delivery stride, the ball, just before release, acquires a height of $110+/-5 \%$ of the bowler's standing height (Salter, Sinclair and Portus 2007). The average height of 26 top ranking fast bowlers was determined to be $1.85+/-0.085 \mathrm{~m}$, which gave a corresponding ball release height of $2.035 \mathrm{~m}$. Utilizing the anthropometric data of Winter (1990), this corresponded to arm, hand and shoulder dimensions of $0.614,0.2$ and $0.479 \mathrm{~m}$ respectively. These arm and hand lengths were comparable to data obtained by Glazier, Paradisis and Cooper (2000) of 0.661 and $0.199 \mathrm{~m}$. The release position of the arm was proposed to be vertical, as suggested by Bartlett, Stockill, Elliott and Burnett (1996) to maximize the delivery length. The evolution of the machine's final dimensions is given in Fig. 2. 


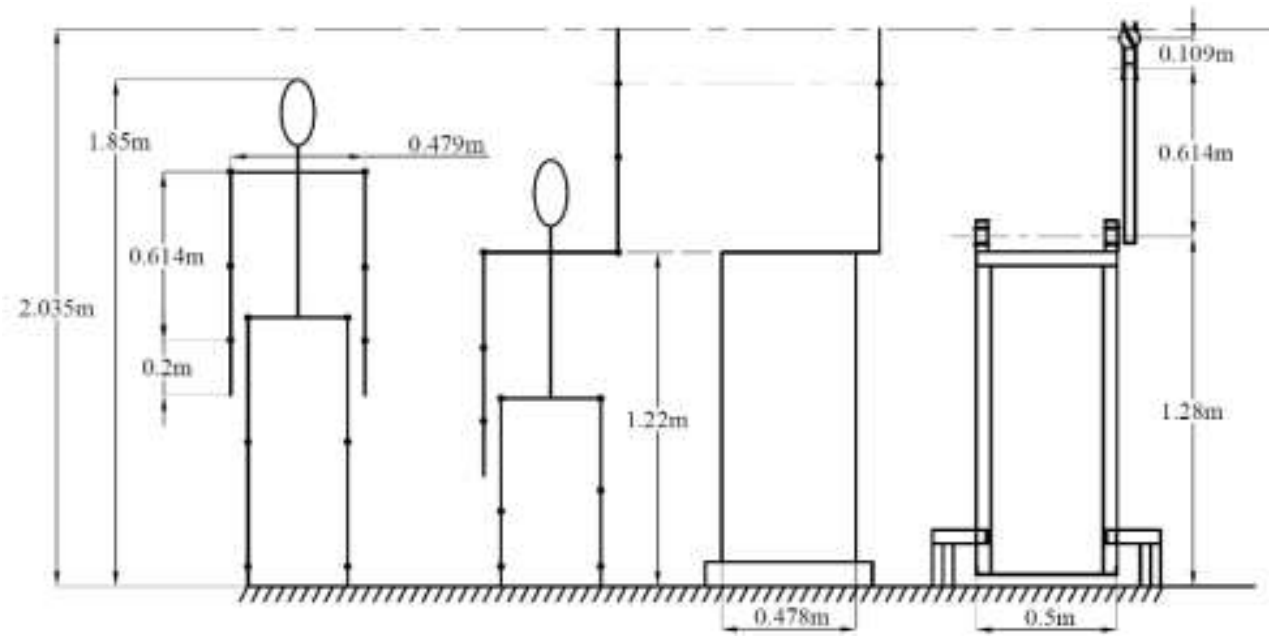

Figure 4: The arm, hand and shoulder lengths for a height of $1.85 \mathrm{~m}$. During ball release, the stride length increases, and the shoulder is lowered. The proposed overall machine dimensions.

\subsection{Actuation and Braking System}

The actuation system influenced the design of the braking, control, transmission and retract systems. In addition to being geometrically constrained to the anthropometric dimensions of the machine, other criteria; manufacturability, operational safety, power transmission, accuracy of mathematical model, power requirements and braking control, needed to be considered. The Analytic Hierarchy Process (AHP) was the decision-making tool employed. Having the highest rank, a linear spring assembly coupled to a shaft via a chain drive was selected as the actuation system. This chain drive extended to a pulley assembly coupled to another linear spring assembly to act as the braking system. The actuation springs are extended to a predetermined distance depending on the desired ball speed by a motor and power screw assembly. A bowling cycle is initiated by the release of this stored potential energy. As the braking springs are engage, the ball is released and the kinetic energy of the arm is stored in the braking springs. The tension in the braking springs are utilized to retract the arm to its initial position.

\subsection{Hand Design}

For fast and medium pace deliveries, the index and middle fingers are placed on either side of the seam, with the thumb on the underneath gently supporting the ball. The initial hand, build by Dr Kelvin Loutan $\mathrm{Jr}$, was redesigned before testing to make it rigid and consistent. Two (2) single fingers were fabricated out of aluminium and bolted directly to the arm with bolts and spacers for strength and stability. The thumb was also spring loaded, and a stopper was added to reduce movement of the thumb.

\subsection{Testing Process}

The testing was done in two (2) phases, that is, testing the bowling machine alone using Pitch Vision software and the second phase was done with batsman facing the bowling machine.

The first phase of testing with the Pitch Vision Software was done with variation of the wrist angle at $-5^{0}$, $0^{0}, 5^{0},-10^{0}, 2.5^{0},-13^{0}$ and $-12.5^{0}$. The speed of the deliveries remained constant at $80 \mathrm{mph}$, which corresponded to a spring extension or plate separation of $780 \mathrm{~mm}$. The Pitch Vision software gave an accurate pitch map and length of each delivery for each wrist angle setting. 
In the second phase of testing, batsmen at different levels, such as minor league, division league, national and international level batted against the bowling machine. Each batsman faced one (1) over or six (6) ball delivered by the machine and then were asked to fill out a simple survey.

\section{Trajectory Model}

For this project, we considered projectile motion of the ball leaving the hand. Then using projectile motion equations, a model equation was derived to predict the path of the cricket ball and to calculate the distance the ball will hit the pitch.

The conditions given for completing the model is as follows:

- Constant launch velocity, $\mathrm{V}=80 \mathrm{mph}(35.7 \mathrm{~m} / \mathrm{s})$.

- Forearm linkage, $1_{1}$ was held at an angle $(\Phi)$ to the horizontal.

- Assumption that the ball was launched at $90^{\circ}$ normal to the hand, $1_{2}$.

Using the equations of motion, the equation used to determine the $\mathrm{y}$ and $\mathrm{x}$ directions were derived;

$$
\begin{aligned}
& y=V_{y} t+\frac{1}{2} g t^{2} \\
& x=V_{x} t
\end{aligned}
$$

Considering the figure below;

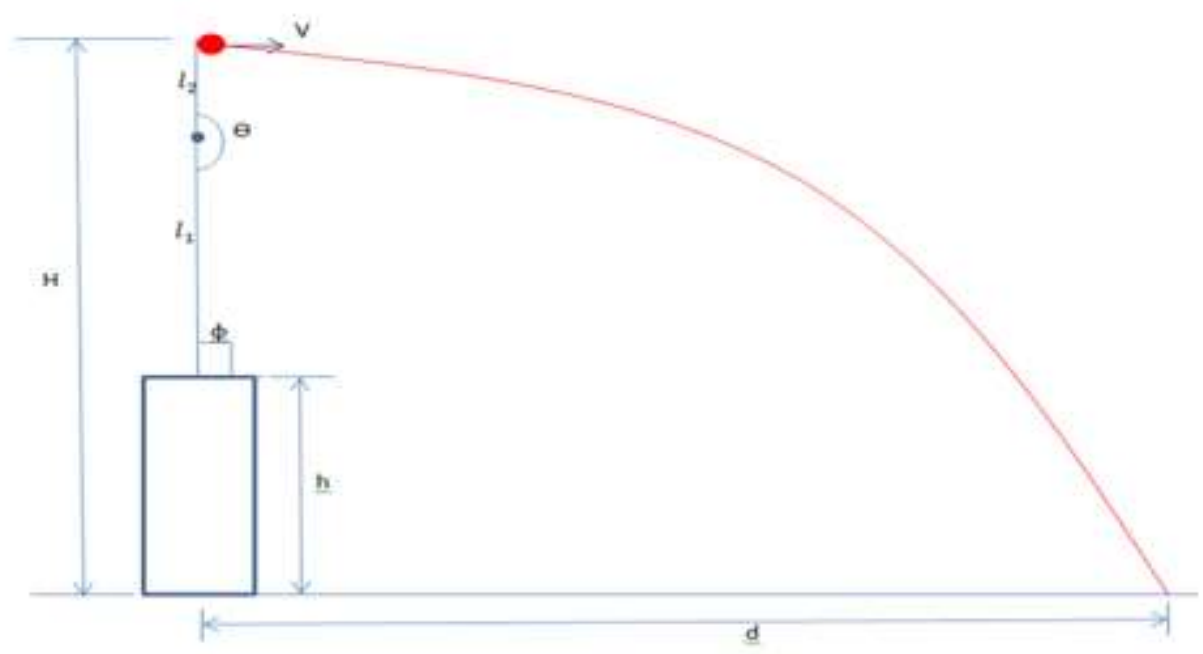

Figure 5: The projected path with the wrist at $0^{0}$ to the vertical.

The velocity in the y axis;

The velocity in the $\mathrm{x}$ axis;

$$
V_{y}=V \sin \propto
$$

The total height before release of ball;

$$
V_{x}=V \cos \propto
$$

$$
H=l_{1}+l_{2}+h
$$

Where $\propto$ is the angle between the y or horizontal axis and the velocity (V) at which the ball is projected. The velocity $(\mathrm{V})$ is projected $90^{\circ}$ from the hand.

Using trigonometry, it was determined that $\alpha=\beta$, where $\beta$ is the wrist angle from the vertical. 
For varying angle of the arm and wrist;

$$
l_{3}=l_{2} \cos \beta
$$

Combining the equations above and using projectile motion theory. The projected distance of the ball can be calculated using the model as follows;

$$
\mathbf{d}=\mathrm{V} \cos \propto\left[\frac{-\mathrm{V} \sin \propto+\sqrt{(\mathrm{V} \sin \propto)^{2}-4\left(\frac{\mathrm{g}}{2}\right)(-\mathrm{H})}}{\mathrm{g}}\right]
$$

Where g, represents gravity.

\subsection{Calculated Values}

The release angle of a human bowler is anywhere between $0^{\circ}$ and $15^{\circ}$ of the vertical, in the forward direction, depending on the type of deliveries being bowled. A snapshot of the release point of the ball, from a video clip, shows that the release angle from the horizontal of the bowling machine, $\Phi$, is $82.03^{\circ}$. This corresponds to a release angle to the vertical of $7.97^{\circ}$ which is in between the range mentioned above. Therefore, for the purpose of the testing and comparison of the data, the release angle would be taken as $82^{\circ}$.

The calculated values for wrist angle and distance were derived from the model equation (7) above and tabulated in Table 1 below. The calculated distance, $\mathrm{d}$, gives you the distance from the bowler's end or the non-striker's end, while the measured values or values obtained from testing were taken from the batsman end or the striker's end. Hence, the calculated distance, d, was subtracted from, the pitch length (L), from stump to stump being 20.12 meters ( 22 yards), to give the distance from the striker's end, D. Thus, we get, $\mathrm{D}=\mathrm{L}-\mathrm{d}$.

Table 1: Table showing wrist angle and distance derived from the model equation.

\begin{tabular}{|l|l|l|}
\hline Wrist Angle, $\boldsymbol{\beta}$ (degrees) & Distance from Bowler, d $(\mathbf{m})$ & Distance from Striker, D $(\mathbf{m})$ \\
\hline-5 & 17.123 & 2.997 \\
\hline-2 & 13.019 & 7.100 \\
\hline 0 & 11.028 & 9.012 \\
\hline 2 & 9.604 & 10.516 \\
\hline 2.5 & 9.137 & 10.983 \\
\hline 3 & 8.821 & 11.299 \\
\hline 5 & 7.722 & 12.398 \\
\hline 7 & 6.829 & 13.291 \\
\hline 9 & 6.096 & 14.024 \\
\hline 10 & 5.777 & 14.343 \\
\hline
\end{tabular}

\section{Results}

The results for the first phase of testing using the Pitch Vision software was done at the Novel Sports Facility. To facilitate these tests, some parameters were kept constant such as, a constant launch velocity of $80 \mathrm{mph}$ (which corresponds to a plate separation of $780 \mathrm{~mm}$ ), the forearm linkage $\left(1_{1}\right)$ was held at $82^{0}$ to horizontal $(\Phi)$ and the assumption that the ball launches at $90^{\circ}$ normal the hand. The wrist angle was varied to get the variation in length. The Table 2 shows the data collected from the Pitch Vision software. 
Table 2: Table showing data taken at varying wrist angle with plate separation of $780 \mathrm{~mm}$.

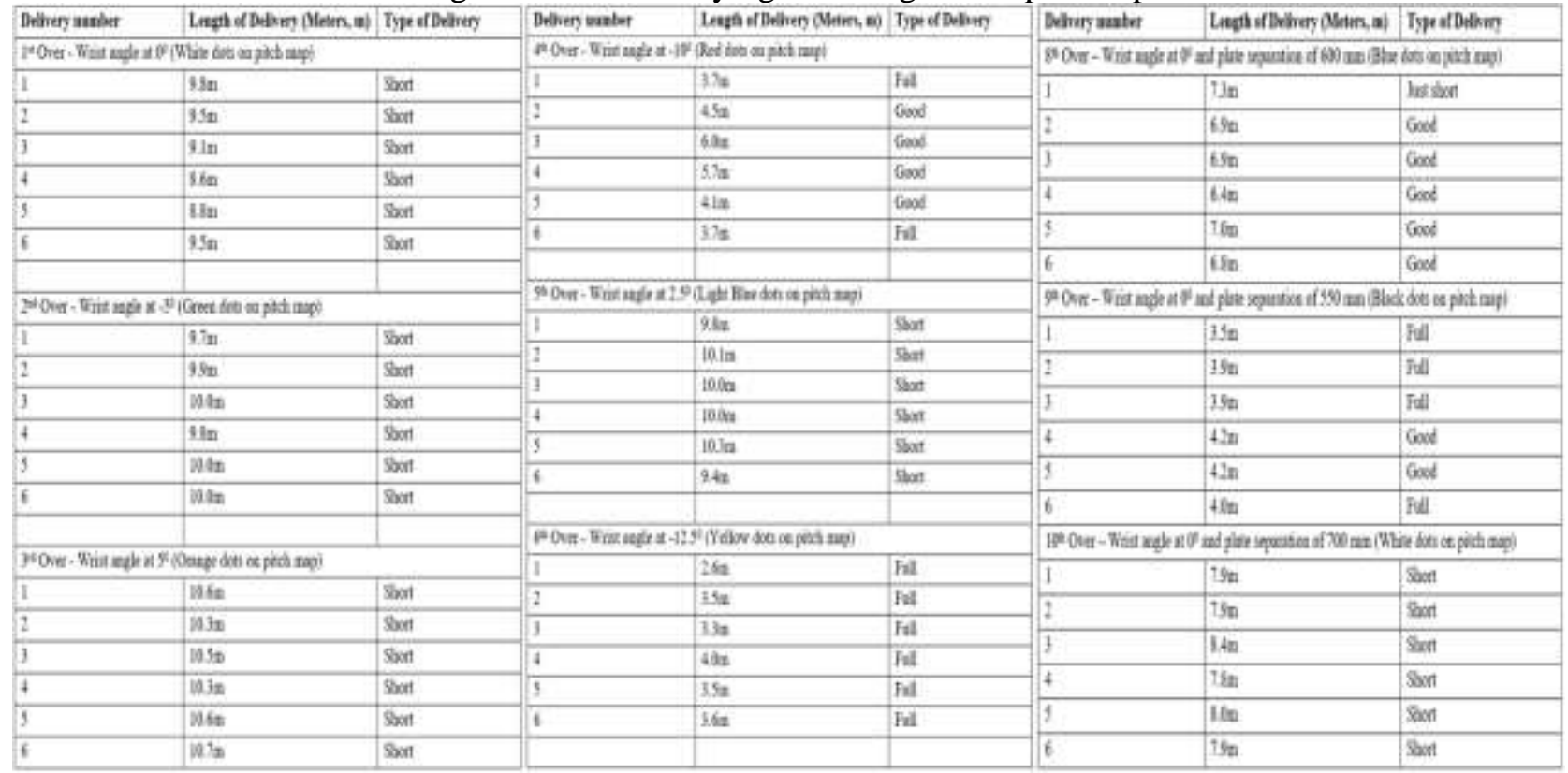

\subsection{Pitch Map}

The pitch map showing the data from the Table 2 above, with each over designated by a different colour and the length of the deliveries indicated by the markings to the bottom of the pitch is seen below.

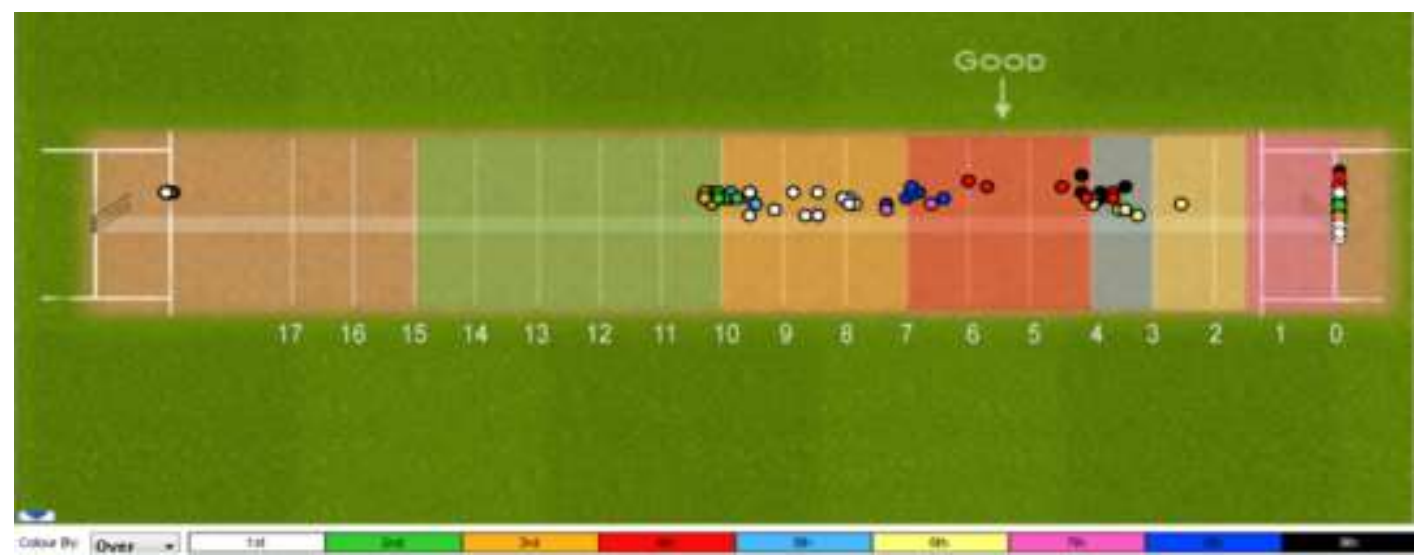

Figure 7: Pitch map of data collected for from Table 2.

\subsection{User Study}

In this study, we look at the coach's and the players perspective. A total of ten (10) batsmen were asked to bat against the machine and fill out a small survey, each batsman faced six (6) ball at varying length and speed. Then five (5) coaches were asked for their views and comments about the bowling machine with the arm and hand. The table below shows the results of the survey.

Table 3: Results of the survey conducted. 


\begin{tabular}{|l|l|l|l|l|l|l|l|}
\hline & \multicolumn{2}{l}{ Feasibility } & \multicolumn{4}{l|}{ Training Aid } & \multicolumn{3}{l|}{ Compared with other Machines } \\
\hline & Feasible & Not & Yes & No & Better & Not Better & Don't know \\
\hline Coach & 5 & 0 & 5 & 0 & 5 & 0 & 0 \\
\hline & & & & & & & \\
\hline & Feasibility & \multicolumn{3}{l|}{ Batsman Set-Up } & \multicolumn{2}{l|}{ Compared with other Machines } \\
\hline & Feasible & Not & Normal & Different & Better & Not Better & Don't know \\
\hline Batsman & 10 & 0 & 10 & 0 & 9 & 0 & 1 \\
\hline & & & & & & & \\
\hline Others & 2 & 0 & & & 2 & 0 & 0 \\
\hline
\end{tabular}

Some of the comments made by the batsmen who faced the machine are; "it's very realistic, since you can follow the ball from the hand", "much better than the traditional machine", "I am really impressed with the machine and its performance", "the ability to follow the arm was clear and clean", "good swift movement which mimic a bowling action", "like how it quick".

\section{Discussion and Conclusions}

\subsection{Scope for Improvement and Further Work}

The testing of the bowling machine has identified several areas for improvement and future work that can advance the design and operation of the machine.

The scope for future work on the bowling machine to make it a commercial product and/or service are as follows:

1. A different method of activation such as using motor instead of the springs.

2. Redesign a more efficient braking system.

3. To design, build and test a new hand with finger and wrist movement as discussed in the final design above.

4. Automation of the bowling machine to enhance its operation, reduce human interaction and render it very user friendly.

5. Redesign the legs and add dampers to stabilise the machine, this reduce the movement of the machine due to the forward force of the arm and spring mechanism.

6. Motorise the wheels and/or redesign the wheel system for ease of movement.

7. To use light weight composite materials for the frame and other parts.

8. To do more field testing, that is, outdoor testing of the bowling machine to test with ideal or real conditions.

\subsection{Comparison of Data}

The experimental values were obtained from the Pitch Vision software during testing, while the calculated values derived from the model equation (Equation 7). The average experimental value was calculated since six (6) values were taken for each wrist angle setting. Table 4 shows the comparison of the data for three (3) wrist angles.

Table 4: The table showing a comparison of Experimental data and Calculated data.

\begin{tabular}{|l|l|l|l|}
\hline Wrist angle, $\beta(\mathrm{deg})$ & $\begin{array}{l}\text { Plate Separation } \\
(\mathrm{mm})\end{array}$ & $\begin{array}{l}\text { Average Experimental } \\
\text { value }(\mathrm{m})\end{array}$ & Calculated Value, D (m) \\
\hline 0 & 780 & 9.217 & 9.012 \\
\hline 2.5 & 780 & 9.933 & 10.983 \\
\hline 5 & 780 & 10.500 & 12.395 \\
\hline
\end{tabular}




\subsection{Summary of Contributions}

A mathematical model predicting the distance the ball pitches after being delivered was developed. The results showed at $0^{0}$ wrist angle the error was at $2.2 \%$ between experimental value and calculated value. At the larger angles the error increased to $9.7 \%$ for a $2.5^{\circ}$ wrist angle and $14.6 \%$ for a wrist angle of $5^{0}$. Hence as the wrist angle increases from the $0^{0}$, if the wrist moves forward or backward, the error increases. This was due to the inaccurate adjustment of the wrist angle. The wrist had no specific setting for the various angles.

The testing procedure was developed to preform specific test on the bowling machine using Pitch Vision to prove that the machine is accurate and repeatability. Looking at the experimental data in the results above the machine is very accurate and reliable as seen in Table 2. For instance, the 2 nd and 3rd over showed a variation in length of 0.4 meters, the 8th over had a variation in length of 0.5 meters and 10th over with a variation of 0.6 meters. The grouping of the deliveries in each over is in and around the one (1) meter mark. A study was done in 2010 to determine how often fast and fast medium bowlers were bowling balls in the channel outside the off-stump (Aneesh Kulkarni, 2010). The results indicated that $73 \%$ of balls bowled were in the channel outside off-stump, and nearly a quarter of balls are bowled short of a good length. The data used to produce those results included all Twenty (20) internationals from June to November 2009, and the 2009 Twenty 20 Champions League. The data included approximately 4800 balls bowled by fast and medium-fast bowlers. Looking at the data collected from Pitch Vision, after testing the bowling machine, showed that of the 78 balls bowled $92.3 \%$ of the balls were outside the off-stump channel. This indicates that the bowling machine is very accurate and repeatable when compared to actual bowlers.

The testing with the batsmen proved that the machine is very functional and valid since it allowed the batsmen to set up or perform their trigger movement as they would in a match and not have to change to suit the traditional bowling machine. The batsmen were able to follow the ball from the hand of the machine all the way to the bat rather than being unsighted for a fraction of time when the ball passes through the traditional bowling machine before being shoot out to the batsmen.

The bowling machine would be able to bowl different types of deliveries with various combinations of wrist angle and plate separation (which correspond to speed). This variation in length and speed could also be achieved by maintaining a fixed wrist angle and varying the plate separation as seen in Table 2 .

In conclusion the evaluation done on the bowling machine with the arm and hand, developed by Dr Kelvin Loutan Jr. at The University of Trinidad and Tobago, with and without a batsman, proves that the machine is usable, functional, repeatable and accurate. Hence, would be a great equipment to assist coaches in their quest to produce the best cricketers. This also proves that the machine can be used for training (working on specific skills or technique) and game situation (making it unpredictable as in a real match). 


\section{References}

[1] P. Persad, N. Ramdass. (2006). To design, build and test a fast bowling robotic arm ((Unpublished bachelor's thesis). The University of The West Indies thesis)

[2] K. Loutan Jr. (2016). To design, fabricate and test an anthropometric fast bowling machine.

[3] Novel Sports (2018). About retrieved from https://www.novelsportstt.com/about/. Multi-sport indoor facility. In https://www.novelsportstt.com/indoor-center/

[4] G. E. Dieter. (2000). Engineering design: A materials and processing approach (3rd ed.). Boston, MA: McGraw-Hill Science/Engineering/Math.

[5] J. E. P. Puig, N. E. N. Rodriguez, M. Ceccarelli. A methodology for the design of robotic hands with multiple fingers. International Journal of Advanced Robotic Systems, 5(2), (2008) 177-184.

[6] C. W. Salter, P. J. Sinclair, M. R. Portus. The associations between fast bowling technique and ball release speed: A pilot study of the within-bowler and between-bowler approaches. Journal of Sports Sciences, 25(11), (2007) 1279-1285.

[7] D. A. Winter. (1990). Biomechanics and motor control of human movement (2nd ed.). New York: Wiley. [8] P. Glazier, G. P. Paradisis, S.-M. Cooper. Anthropometric and kinematic influences on release speed in men's fast-medium bowling. Journal of Sports Sciences, 18(12), (2000) 1013-1021.

[9] R. M. Bartlett, N. P. Stockill, B. C. Elliott, A. F. Burnett. The biomechanics of fast bowling in men's cricket: A review. Journal of Sports Sciences, 14(5), (1996) 403-424.

[10] R. Bickramdass. (2019). Cricket Coaching Assistant. ((Unpublished Master's thesis). The University of Trinidad and Tobago thesis).

[11] The Physics of Projectile Motion. In https://www.mansfieldct.org/Schools/MMS/staff/hand/Projectilemotion.htm

[12] A. Kulkarni. (2010). Scoring Variation by Line \& Length of the Bowler's Delivery. Against the spin. https://againstthespin.com/scoring-variation-by-line-length-of-the-bowlers-delivery-c77b385332ca 